\title{
PASSIVE END POLE COMPENSATION SCHEME FOR A 1.8 TESLA WIGGLER
}

\author{
L.H.Chang, Ch.Wang, C.H.Chang, T.C.Fan \\ Synchrotron Radiation Research Center, Hsinchu, Taiwan
}

\begin{abstract}
An optimized end pole compensation scheme for the 1.8 Tesla, 25-pole wiggler W20/SRRC was studied by using 2D magnetostatic code OPERA-2d. The maximum deviation of the dipole steering for this wiggler can be minimized either by optimizing the sizes of the bias magnets or by optimizing the easy-axis rotating angles of the permanent rotators. After optimization, the dipole steering is within 100 Gauss-cm in the operating gap range so that a passive compensation scheme is possible even for an insertion device configured with very strong on-axis magnetic field strength.
\end{abstract}

\section{INTRODUCTION}

There are usually end correctors located at both ends of the insertion device to compensate the residual dipole steering due to the fringe magnetic field contributions as well as the magnetic field errors, which can be characterized by the first integral of the magnetic field $\left(I_{1}\right)$ through the entire magnetic structure. Basically, the design goal of the end correction scheme could be guided in two directions: passive correction and compact size. A passive end correction scheme means that the maximum deviation of the dipole steering between the minimum and maximum gap range can be within a reasonable small value like 100 Gauss-cm without help of any gap-dependent active compensation mechanism. Recess of the next-to-last pole pieces[1] demonstrates one of the solutions. However, optimization of the size of the permanent bias magnets in the end correction configuration gives another possibility.

The hybrid permanent periodic magnetic structure of the STI delivered 1.8 Tesla, 25-pole wiggler W20/SRRC was used as an example for numerical study of this possibility in this report. The magnetostatic code OPERA-2d[2] was used. A Halbach rotator type end pole compensation scheme[3] composes of two sets of permanent bias magnets (1 and 2) and rotators ( $\alpha$ and $\beta$ ) located between pole 0 (field clamp) and pole 2 , as shown in Fig. 1.

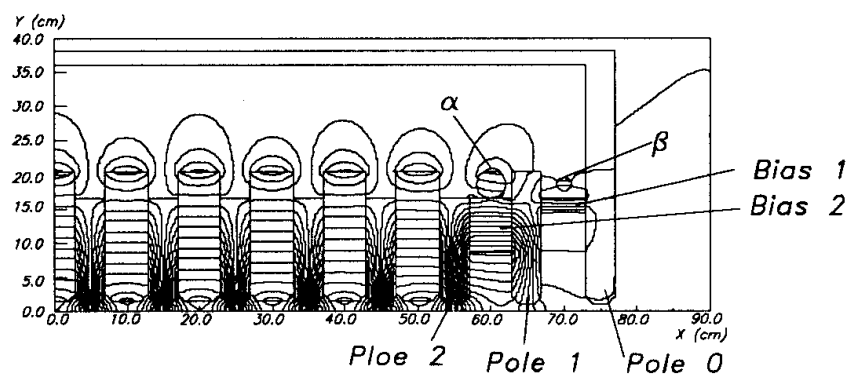

Figure 1. 2D numerical modelling of the new proposed end correctors for the wiggler W20/SRRC

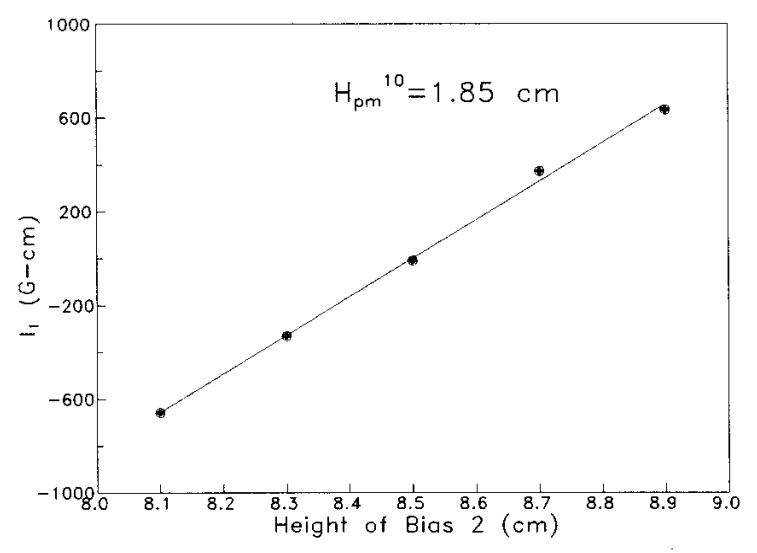

Figure 2. Dipole steering $I_{1}$ at $22 \mathrm{~mm}$ gap as a function of the height of the bias magnet 2 . The height of bias magnet 1 is equal to $1.85 \mathrm{~cm}$.

\section{OPTIMIZATION OF BIAS MAGNETS}

The maximum deviation of the first integral of the magnetic field $\left(\Delta I_{1}\right)$ through the entire insertion device is strongly dependent on the size of the bias magnets. With a fixed size of the bias magnet 1 , the calculated dipole steering at a fixed gap (here 22 mm gap) is proportional to the height of the bias magnet 2, as shown in Fig. 2. On the other hand, with a fixed size of the bias magnet 2 , the calculated dipole steering at a fixed gap as a function of the height of the bias magnet 1 has a negative slope, as shown in Fig. 3. Fig. 4 shows the maximum deviation of the dipole steering in the gap range from $22 \mathrm{~mm}$ to $102 \mathrm{~mm}$ as a function of the height of the bias magnet 2 . Here the heights of the bias magnet 1 was optimized so that the dipole steering always keeps zero at $22 \mathrm{~mm}$ gap.

With fixed heights of the bias magnets, the dipole steering is usually gap-dependent, as shown in Fig. 5. The maximum deviation of the dipole steering could be huge, if the dimension of the bias magnets is not properly optimized. For example, the maximum deviation of the dipole steering is more than 1500 Gauss-cm, if the height of the bias magnet 2 is equal to $9.5 \mathrm{~cm}$. However, the maximum dipole steering deviation is within 100 Gauss-cm, if the height of the bias magnet 2 is equal to $7.95 \mathrm{~cm}$. In Fig. 5, the heights of the bias magnet 1 were optimized to keep zero dipole steering at $22 \mathrm{~mm}$ gap, i.e. the heights of the bias magnet 2 are equal to be $7.95 \mathrm{~cm}$ and $9.50 \mathrm{~cm}$ individually.

After comparison between the results shown in the Fig. 2 and Fig. 4, we found that the maximum deviation of the dipole steering in the gap range from $22 \mathrm{~mm}$ to $102 \mathrm{~mm}$ can be minimized by reducing the height of the bias magnet 2 to about $7.95 \mathrm{~cm}$.

\section{FINE TUNE WITH ROTATORS}

A discrepancy between the simulated and measured dipole steering for a given end correction scheme is The unavoidable 


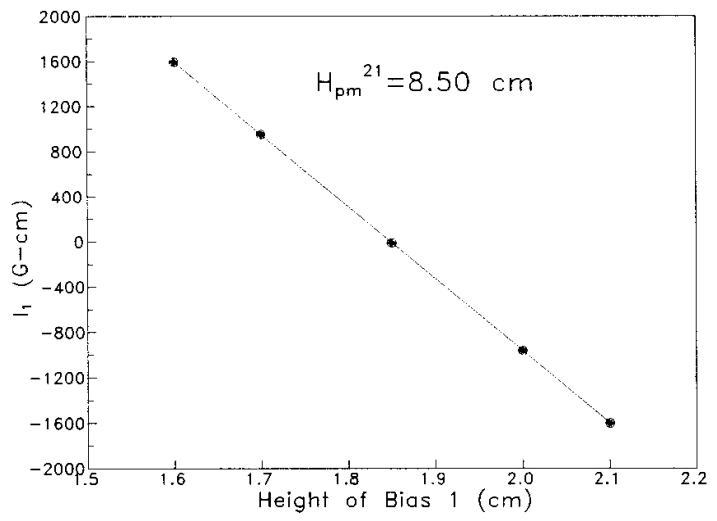

Figure 3. Dipole steering $I_{1}$ at $22 \mathrm{~mm}$ gap as a function of the height of bias magnet 1 at $22 \mathrm{~mm}$ gap. The height of bias magnet 2 is equal to $8.5 \mathrm{~cm}$.

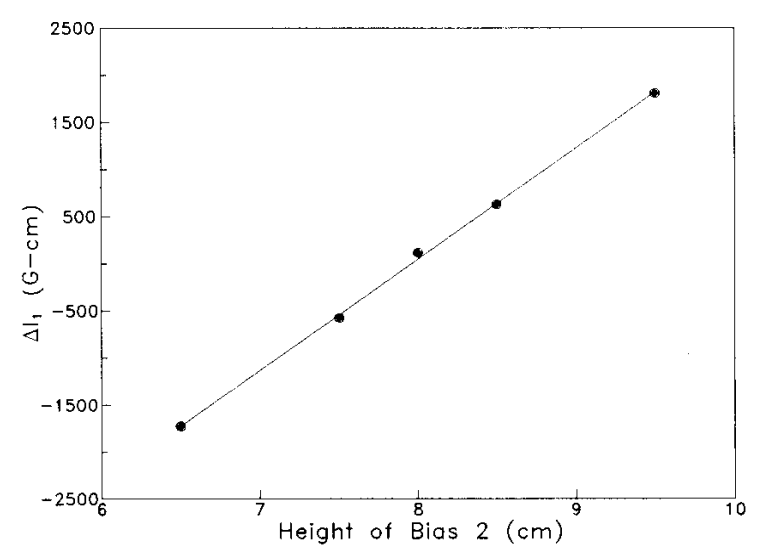

Figure 4. Maximum deviation of the dipole steering $\Delta I_{1}$ in the gap range from $22 \mathrm{~mm}$ to $102 \mathrm{~mm}$ as a function of the height of bias magnet 2 .

magnetic field errors and $3 \mathrm{~d}$ effect due to finite width of the magnetic structure are two of the most possible sources for this discrepancy. A mechanism for fine tuning of the dipole steering after assembly of the insertion device magnetic structure is therefore necessary. There are two tunable permanent magnetic rotators equipped in the Halbach rotator type end pole scheme for this purpose (see Fig. 1). However, the rotators could be replaced by electromagnetic coils for some other reasons.

As shown in Fig. 6, a pair of the permanent rotators with steering tuning capability larger than \pm 3000 Gauss-cm for the wiggler W20/SRRC at $22 \mathrm{~mm}$ gap was used for our preliminary numerical study. Here the radius of the rotators are $2.0 \mathrm{~cm}$ and $1.0 \mathrm{~cm}$ individually. At a given gap, the dipole steering can be completely compensated by rotating either the rotator $\alpha$ or $\beta$. In Fig. 7, the maximum deviation of the dipole steering is shown as a function of the easy-axis rotating angle of the permanent rotator $\alpha$, with fixed rotating angle of the rotator $\beta$ such that the dipole steering keeps zero at $22 \mathrm{~mm}$ gap. In Fig. 8, the dipole steering as a function of gap is shown. With help of the rotators, the residual dipole steering can be within 100 Gauss-cm without difficulty after optimization of the easy-axis rotating angles of both rotators. On comparison of the results shown in Fig. 7 and 8 with those shown in Fig. 4 and 5, we found that to optimize

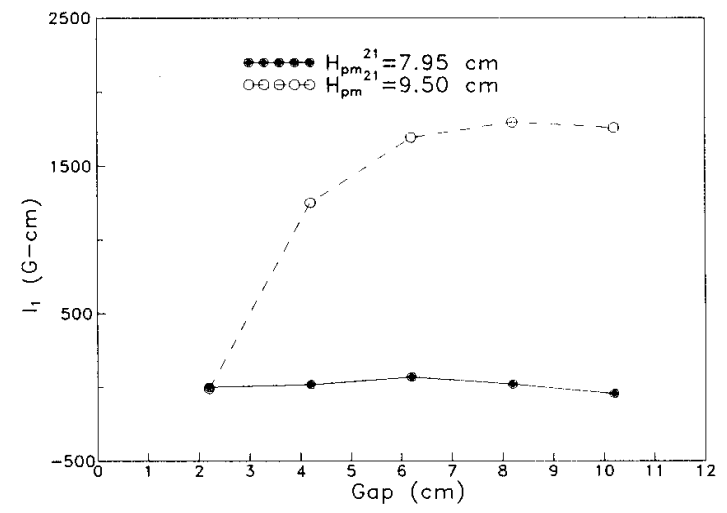

Figure 5. Dipole steering as a function of gap for the heights of the bias magnet 2 equal to $7.95 \mathrm{~cm}$ and $9.50 \mathrm{~cm}$.

the size of the bias magnets is similar to optimize the rotating angles of the permanent rotators.

\section{DISCUSSIONS}

The maximum deviation of the dipole steering in the operating gap range of an insertion device can be achieved either by optimizing the heights of bias magnets or by optimizing the easy-axis rotating angles of the permanent rotators, if a Halbach rotator type end pole compensation scheme is to be used. Numerically, it is possibly to have a maximum deviation of the dipole steering within 100 Gauss-cm with each approaches. Therefore, a passive end compensation scheme can be achieved with help of prior optimization of the heights of bias magnets by using magnetostatic code and with fine tune of the easy-axis rotating angles of the permanent rotators after assembly of the magnetic structure of the insertion device.

\section{References}

[1] I.Vasserman and E.R. Moog, "A Passive Scheme for ID End Correction,", presented at the International Conference on Synchrotron Radiation Instrumentation, held 18-22, July, 1994 New York, USA; I.Vasserman and E.R. Moog, ”A Passive Scheme for Undulator End Correction," Appendix 2 of the Technical Specification for Undulator A, APS Document No. 41010101-00002.

[2] OPERA-2d is a trade mark of Vector Fields.

[3] U5.0 Undulator Conceptual Design Report, LBL PUB5256, November 1989. 


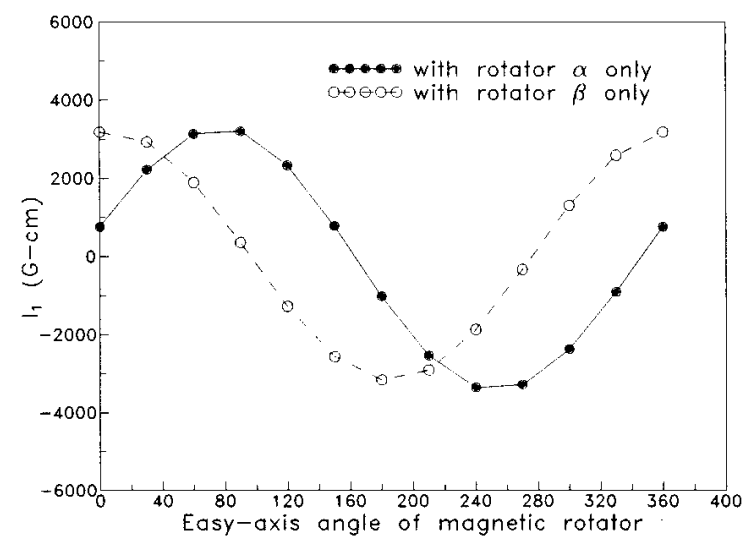

Figure 6. Dipole steering as a function of the easy-axis rotating angle of the permanent magnet.

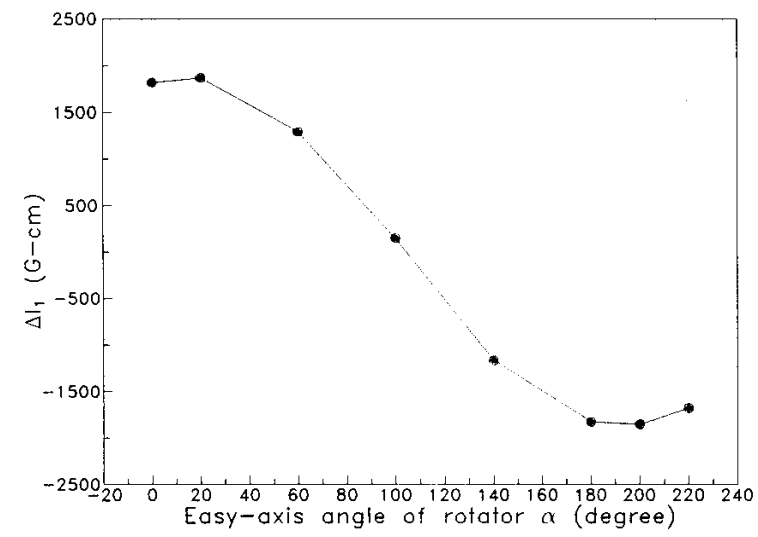

Figure 7. Maximum deviation of the dipole steering $\Delta I_{1}$ in the gap range from $22 \mathrm{~mm}$ to $102 \mathrm{~mm}$ as a function of the easy-axis rotating angle of the permanent rotator $\alpha$.

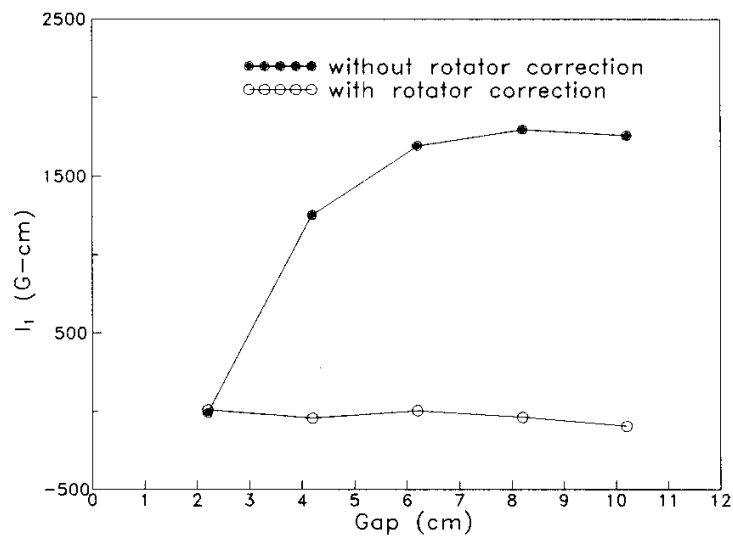

Figure 8. Dipole steering as a function of gap for an end correction scheme with (solid curve) and without (dashed curve) help of the rotators. 